\section{Molecular Syndromology}

Mol Syndromol 2017;8:115-117

DOI: $10.1159 / 000454964$
Accepted: November 30, 2016

by M. Schmid

Published online: January 14, 2017

\title{
Of Simple and Complex Genome Rearrangements, Chromothripsis, Chromoanasynthesis, and Chromosome Chaos
}

The genomes of all eukaryotes are contained within a set of chromosomes with a, for each species characteristic, number and appearance collectively known as the karyotype. Alterations in this karyotype, either occurring in the germline, in dividing somatic cells, or in postmitotic cells, are often associated with considerable phenotypic effects [Poot and Haaf, 2015]. The mere complexity of a karyotypic alteration, be it a simple copy number variation (CNV) or a complex chromosome rearrangement (CCR), is not a measure of its possible phenotypic effect; approximately $70 \%$ of all CCRs proved not to be associated with any clinical phenotype at all [Pellestor et al., 2011]. Among the documented phenotypic mechanisms of CCRs are gene dosage-dependent effects and disruptions of chromosomal architecture, producing truncated and de novo fused genes or affecting topologically associated domains [Poot and Haaf, 2015; Lupiáñez et al., 2016; Spielmann and Mundlos, 2016]. In cultured tumor cells, treatments with drugs such as doxorubicin and mitomycin $\mathrm{C}$ result in massive chromosome fragmentation and cell death [Liu et al., 2014]. The surviving cells show "chaotic" karyotypes in which numerous fragments from several chromosomes at once are stitched together by nonhomologous end joining (NHEJ) in a seemingly random process [Liu et al., 2014; Giam and Rancati, 2015]. This appears not to be a single catastrophe, but rather an ongoing process leading to unprecedented genome instability reflected by nonclonal chromosome rearrangements and increased transcriptome dynamics [Stephens et al., 2012; Heng et al., 2016]. Such chromosome fragmentation,

\section{KARGER}

๑๑ 2017 S. Karger AG, Basel

E-Mail karger@karger.com

www.karger.com/msy known as "chromothripsis", has been observed in cells that are either deficient in $\mathrm{p} 53$ or in which its function has been experimentally disabled [Rausch et al., 2012; Mardin et al., 2015; Zhang et al., 2015]. Chromothripsis is currently defined as a process in which one or several chromosomes are broken into many segments with narrowly spaced breakpoints, subsequently being joined in random order and orientation, whereby some fragments are lost and others preserved, such that an alternating pattern of lost and retained heterozygosity emerges [Holland and Cleveland, 2012; Korbel and Campbell, 2013; Zhang et al., 2013; Nazaryan-Petersen and Tommerup, 2016]. While chromothripsis may occur repeatedly in growing tumor cells, it appears as a single cataclysmic event in the germline [Kloosterman et al., 2011, 2012; Nazaryan et al., 2014; Nazaryan-Petersen and Tommerup, 2016]. Chromothripsis may result from under-replication of one or several chromosomes being temporarily isolated in a micronucleus or from a telomere crisis followed by breakage-fusion-bridge cycles [Zhang et al., 2013, 2015; Maciejowski et al., 2015; Mardin et al., 2015]. Alternatively, a chromothripsis-like pattern, typified by multiple CNVs including deletions, duplications, and/or triplications as well as translocations and inversions, may arise in the germline [Liu et al., 2011]. Breakpoint sequencing of these cases revealed small templated insertions and microhomology at the breakpoint junctions, which have been attributed to DNA replication errors, such as fork stalling and template switching and microhomology-mediated break-induced replication [Liu et al., 2011]. Thus,

Martin Poot

Department of Human Genetics, University of Würzburg Biozentrum, Am Hubland DE-97074 Würzburg (Germany)

E-Mail Martin_Poot@hotmail.com 
this process of chromoanasynthesis, i.e., chromosome reconstitution, results in clustered CNVs, including deletions, duplications, and triplications [Liu et al., 2011]. Recently, 3 cases of chromoanasynthesis with rearrangements of a single chromosome involving duplications and insertion translocations as well as a novel cell-division independent mechanism of chromothripsis have been reported [Masset et al., 2016; Nazaryan-Petersen et al., 2016].

Using array-CGH screening, 3 patients with 8-11 copy number gains dispersed across a single chromosome were found [Masset et al., 2016]. By FISH analyses, the copy number gains proved to be insertion-duplications of DNA segments at distant locations within the same chromosome. The insertion breakpoint junctions showed microhomologies and nontemplated insertions of up to 40 bp. The latter finding cannot be reconciled with classical NHEJ. On the other hand, the highly dispersed nature of the rearrangements cannot be accounted for by assuming a single fork stalling and template-switching event during DNA replication [Liu et al., 2011; Masset et al., 2016]. Since no triplications and quadruplications were found, these cases are clearly distinct from the homology-driven DNA repair mechanism mediated by multiple template switches recently documented for the PLP1 locus [Beck et al., 2015]. Therefore, the authors invoke an alternative NHEJ mechanism, probably mediated by DNA Pol $\theta$, to account for the CCRs in their cases [Masset et al., 2016].

In 11 individuals of a 3-generation family, a germline chromothripsis event involving 6 breaks in chromosome arm $3 q$ and 1 break in $5 q$ was reported [Bertelsen et al., 2016; Nazaryan-Petersen et al., 2016]. At 2 breakpointjoining sites, no homologies, deletions, insertions, or inversions were found, which is consistent with NHEJ depending upon the activities of the Ku70/80 heterodimer and the DNA ligase IV/XRCC4 complex [Lieber, 2010]. In a second pair of breakpoint-joining sites, involving a paracentric inversion, 2 very short microhomologies were detected, which indicates that these breakpoints were ligated independent of Ku70/80 and DNA ligaseIV activities by microhomology-mediated break-induced replication. The remaining 2 breakpoint-joining sites harbor a 177-bp homeology and an inserted retrotransposon, which have not previously been reported in cases of chromothripsis. The authors hypothesize that the Alu elements residing at 4 breakpoints may have triggered the formation of 2 large hairpin loops, which then facilitated physical interactions between distally located DNA segments on chromosome arms $3 \mathrm{q}$ and $5 \mathrm{q}$. In agreement with Alu/Alu recombination-mediated double-strand break repair is the presence of inverted Alu elements (AluSq and AluJb) flanking a pair of breakpoints. Thus, L1 endonuclease activity may have produced several DNA breaks and mediated retrotransposition of SINEVNTR-Alu elements in close proximity with a deletion. L1 endonuclease and SINE-VNTR-Alu elements are expressed in human germinal vesicles and de novo retrotransposition events have been found in human primary oocytes [Georgiou et al., 2009]. While this is the first report of a chromothripsis event involving L1 endonuclease-mediated retrotransposition, some 20 simple pathogenic rearrangements have been reported, of which 17 were associated with deletions at the insertion site ranging from $1.4 \mathrm{~kb}$ to $1 \mathrm{Mb}$ in size. These cases shared 4 characteristics with the present chromothripsis event. First, all insertions occured at L1 endonuclease cleavage sites. Second, the $5^{\prime}$-ends of almost all insertions (15 out of 17) are extremely truncated with some having only poly-A tails remaining. Third, large deletions always occur at the $5^{\prime}$ end of the insertion. Fourth, none of the cases showed duplications at their target sites. Thus, retrotransposition-mediated chromothripsis and $\mathrm{CNV}$ formation represent a replication and micronucleus-independent mechanism of chromosome rearrangements, not limited to the germline, which may also produce somatic deletion mosaicism in the brain [Erwin et al., 2016].

Thus far, little is known regarding the underlying mechanism(s) that may precipitate micronucleus- or retrotranspostion-mediated processes of chromosome shattering that result in either cell death or chromothripsis [Nazaryan-Petersen and Tommerup, 2016]. Interesting$\mathrm{ly}$, the distribution of chromosomes involved in germline chromothripsis is not random. In 22 reports of germline chromothripsis, 62 chromosomes were affected, of which chromosome 5 was the most often involved (8 times), followed by chromosome 7 ( 6 times), and 3 as well as 9 (5 times each) [Nazaryan-Petersen and Tommerup, 2016]. By eye, this distribution differs from those of single and multiple small supernumerary marker chromosomes [Hochstenbach et al., in press]. This suggests that the mechanisms leading to formation of small supernumerary marker chromosomes and chromothripsis may be distinct. However, more elaborate studies of chromosome distributions in micronuclei produced by precisely defined molecular mechanisms are needed to support or to reject some of the proposed putative causes of chromothripsis [Pellestor et al., 2014; Hovhannisyan et al., 2016; Nazaryan-Petersen and Tommerup, 2016].

Martin Poot
116

Mol Syndromol 2017;8:115-117 DOI: $10.1159 / 000454964$
Poot 


\section{References}

Beck CR, Carvalho CM, Banser L, Gambin T, Stubbolo D, et al: Complex genomic rearrangements at the PLP1 locus include triplication and quadruplication. PLoS Genet 11:e1005050 (2015).

Bertelsen B, Nazaryan-Petersen L, Sun W, Mehrjouy MM, Xie G, et al: A germline chromothripsis event stably segregating in 11 individuals through three generations. Genet Med 18:494-500 (2016).

Erwin JA, Paquola AC, Singer T, Gallina I, Novotny $\mathrm{M}$, et al: $\mathrm{L} 1$-associated genomic regions are deleted in somatic cells of the healthy human brain. Nat Neurosci 19:1583-1591 (2016).

Georgiou I, Noutsopoulos D, Dimitriadou E, Markopoulos G, Apergi A, et al: Retrotransposon RNA expression and evidence for retrotransposition events in human oocytes. Hum Mol Genet 18:1221-1228 (2009).

Giam M, Rancati G: Aneuploidy and chromosomal instability in cancer: a jackpot to chaos. Cell Div 10:3 (2015).

Heng HH, Regan SM, Liu G, Ye CJ: Why it is crucial to analyze non clonal chromosome aberrations or NCCAs? Mol Cytogenet 9:15 (2016).

Hochstenbach R, Poot M, Liehr T: Mechanisms of origin and clinical effects of multiple small supernumerary marker chromosomes, each derived from a different chromosome. OBM Genomics, in press (2017).

Holland AJ, Cleveland DW: Chromoanagenesis and cancer: mechanisms and consequences of localized, complex chromosomal rearrangements. Nat Med 18:1630-1638 (2012).

Hovhannisyan G, Aroutiounian R, Babayan N, Harutyunyan T, Liehr T: Comparative analysis of individual chromosome involvement in micronuclei induced by mitomycin $\mathrm{C}$ and bleomycin in human leukocytes. Mol Cytogenet 9:49 (2016).
Kloosterman WP, Guryev V, van Roosmalen M, Duran KJ, de Bruijn E, et al: Chromothripsis as a mechanism driving complex de novo structural rearrangements in the germline. Hum Mol Genet 20:1916-1924 (2011).

Kloosterman WP, Tavakoli-Yaraki M, van Roosmalen MJ, van Binsbergen E, Renkens I, et al: Constitutional chromothripsis rearrangements involve clustered double-stranded DNA breaks and nonhomologous repair mechanisms. Cell Rep 1:648-655 (2012).

Korbel JO, Campbell PJ: Criteria for inference of chromothripsis in cancer genomes. Cell 152: 1226-1236 (2013)

Lieber MR: The mechanism of double-strand DNA break repair by the nonhomologous DNA end-joining pathway. Annu Rev Biochem 79:181-211 (2010).

Liu G, Stevens JB, Horne SD, Abdallah BY, Ye KJ, et al: Genome chaos: survival strategy during crisis. Cell Cycle 13:528-537 (2014).

Liu P, Erez A, Nagamani SC, Dhar SU, Kołodziejska KE, et al: Chromosome catastrophes involve replication mechanisms generating complex genomic rearrangements. Cell 146:889-903 (2011).

Lupiáñez DG, Spielmann M, Mundlos S: Breaking TADs: how alterations of chromatin domains result in disease. Trends Genet 32:225237 (2016).

Maciejowski J, Li Y, Bosco N, Campbell PJ, de Lange T: Chromothripsis and kataegis induced by telomere crisis. Cell 163:1641-1654 (2015).

Mardin BR, Drainas AP, Waszak SM, Weischenfeldt J, Isokane $M$, et al: A cell-based model system links chromothripsis with hyperploidy. Mol Syst Biol 11:828 (2015).

Masset H, Hestand MS, Van Esch H, Kleinfinger P, Plaisancié J, et al: A distinct class of chromoanagenesis events characterized by focal copy number gains. Hum Mutat 37:661-668 (2016).
Nazaryan L, Stefanou EG, Hansen C, Kosyakova N, Bak M, et al: The strength of combined cytogenetic and mate-pair sequencing techniques illustrated by a germline chromothripsis rearrangement involving FOXP2. Eur J Hum Genet 22:338-3443 (2014).

Nazayran-Petersen L, Tommerup N: Chromothripsis and human genetic disease. eLS 1-10 (2016).

Nazaryan-Petersen L, Bertelsen B, Bak M, Jønson L, Tommerup N, et al: Germline chromothripsis driven by L1-mediated retrotransposition and Alu/Alu homologous recombination. Hum Mutat 37:385-395 (2016).

Pellestor F, Anahory T, Lefort G, Puechberty J, Liehr T, et al: Complex chromosomal rearrangements: origin and meiotic behavior. Hum Reprod Update 17:476-494 (2011).

Pellestor F, Gatinois V, Puechberty J, Geneviève D, Lefort G: Chromothripsis: potential origin in gametogenesis and preimplantation cell divisions. A review. Fertil Steril 102:1785-1796 (2014).

Poot M, Haaf T: Mechanisms of origin, phenotypic effects and diagnostic implications of complex chromosome rearrangements. Mol Syndromol 6:110-134 (2015).

Rausch T, Jones DT, Zapatka M, Stütz AM, Zichner $\mathrm{T}$, et al: Genome sequencing of pediatric medulloblastoma links catastrophic DNA rearrangements with TP53 mutations. Cell 148: 59-71 (2012).

Spielmann M, Mundlos S: Looking beyond the genes: the role of non-coding variants in human disease. Hum Mol Genet 25:R157-165 (2016).

Stevens JB, Liu G, Abdallah BY, Horne SD, Ye KJ, et al: Unstable genomes elevate transcriptome dynamics. Int J Cancer 134:2074-2087 (2014).

Zhang CZ, Leibowitz ML, Pellman D: Chromothripsis and beyond: rapid genome evolution from complex chromosomal rearrangements. Genes Dev 27:2513-2530 (2013).

Zhang CZ, Spektor A, Cornils H, Francis JM, Jackson EK, et al: Chromothripsis from DNA damage in micronuclei. Nature 522:179-184 (2015). 